\title{
ÍNDICE DE COBERTURA VEGETAL E SUA MODELAGEM PARA CULTIVARES DE SOJA NO SUL DE MINAS GERAIS
}

\section{Index of vegetal cover and its modeling for soybean cultivars in the south of Minas Gerais}

\author{
Vitor Corrêa de Mattos Barreto ${ }^{1}$, Marx Leandro Naves Silva ${ }^{2}$, Nilton Curi', \\ Flávio Pereira de Oliveira ${ }^{4}$, Pedro Milanez de Rezende ${ }^{5}$, Daniel Ferreira Furtado ${ }^{6}$
}

\begin{abstract}
RESUMO
A cobertura vegetal do solo é decisiva para redução dos efeitos erosivos do impacto direto das gotas de chuva na superfície do solo. Desta forma, objetivou-se com este estudo determinar o índice de cobertura vegetal (CV) e desenvolver modelos para sua estimativa para a cultura da soja, usando os atributos climáticos no período de chuvas intensas no Sul de Minas Gerais. As determinações da CV foram feitas semanalmente, na área experimental do Departamento de Ciência do Solo, Universidade Federal de Lavras, no período de novembro de 1999 a maio de 2000, em 28 cultivares de soja com potencial para cultivo nesta região. Para avaliação da cobertura vegetal foi utilizada a metodologia descrita por Stocking (1988). Na modelagem procurou-se relacionar a CV com os valores acumulados dos seguintes atributos climáticos: temperatura média ( $\mathrm{T}_{\text {med }}$ ), precipitação (PREC) e umidade relativa do ar (UR). Os valores de cobertura vegetal apresentaram uma amplitude de variação de 56 a 83\%, sendo BR 162, LO 12 L e M. Soy 108 as cultivares mais eficientes e FT Abyara e Tucano as menos eficientes. O hábito diferencial de crescimento das cultivares ajuda a explicar esse comportamento. O modelo ajustado adequado para estimativa da CV foi: $\mathrm{CV}=116589,976+0,422$. Tmed $+0,132$. PREC - 0,095 . UR + 0,000024. Tmed ${ }^{2}, R^{2}=0,99(P<0,01)$. A determinação da CV nas diferentes fases de desenvolvimento da cultura é de grande importância, uma vez que seu estabelecimento coincide com o período de maior potencial erosivo das chuvas na região estudada.
\end{abstract}

Termos para indexação: Área foliar, proteção do solo, Glycine max (L.) Merr., estimativa, taxa de crescimento, características agronômicas.

\begin{abstract}
Vegetal cover of soil is decisive to reduce the erosive effects of direct impact of raindrops on the soil surface. Therefore, the objective of this study was to determine the vegetal cover (CC) index and to develop models to estimate it for soybean cultivars, using climatic attributes in the period of intense rains in the South of the State of Minas Gerais in Brazil. CC was measured weekly in the experimental area of the Department of Soil Science, Federal University of Lavras, from November 1999 to May 2000 , for 28 soybean cultivars with yield potential in this region. To evaluate the vegetal cover, the method described by Stocking (1988) was used. In the modeling, CC was related with the accumulated values of following climatic attributes: medium temperature (Tmed), precipitation (PREC), and relative humidity of the air (RH). Vegetal cover values presented an amplitude from 56 to $83 \%$, being BR 162, LO $12 \mathrm{~L}$ and M. Soy 108, the more efficient cultivars, and the FT Abyara and Tucano, the least efficient ones. The differential growth habit of the cultivars helps to explain this behavior. The best adjusted model for the estimative of CC was: $\mathrm{CC}=116589.976$ +0.422 , Tmed + 0.132, PREC - 0.095, RH + $0.000024 \mathrm{Tmed}^{2}, \mathrm{R}^{2}=0.99(\mathrm{P}<0.01)$. The knowledge of CC for different development phases of the crop is of great importance, taking into account that its establishment time coincides with the highest erosive potential of the rains in the studied region.
\end{abstract}

Index terms: Leaf area, soil cover, Glycine $\max ($ L.) Merr., modeling, growth rate, agronomic characteristics.

(Recebido em 24 de outubro de 2007 e aprovado em 14 de abril de 2009)

\section{INTRODUÇÃO}

A erosão hídrica é um dos fatores de maior degradação do solo. Sua ação representa danos econômicos e ambientais, podendo gerar sérios problemas sociais.

A cobertura do solo, isoladamente, é o fator de maior importância relativa no controle da erosão hídrica. A utilização dos resíduos culturais como cobertura do solo é uma maneira simples, eficaz e econômica de controlar a erosão nas áreas cultivadas. Os resíduos, quando mantidos sobre a superfície do solo, evitam o impacto direto das gotas da chuva, prevenindo a desagregação e o selamento superficial e mantendo taxas adequadas de infiltração de água no solo (Amado et al., 1989).

\footnotetext{
1Universidade Estadual Paulista Júlio de Mesquita Filho/UNESP - Departamento de Produção Vegetal - Jaboticabal, SP 2Universidade Federal de Lavras/UFLA - Departamento de Ciência do Solo/ DCS - Cx. P. 3037 - 37200-000 - Lavras, MG - marx@ufla.br 3Universidade Federal de Lavras/UFLA - Departamento de Ciência do Solo/ DCS - Lavras, MG

${ }^{4}$ Universidade Federal do Rio Grande do Sul/UFRGS - Porto Alegre, RS

${ }^{5}$ Universidade Federal de Lavras/UFLA - Departamento de Agricultura/ DAG - Lavras, MG

Guniversidade Federal de Lavras/UFLA - Departamento de Ciências Exatas/ DEX - Lavras, MG
} 
Atualmente, no Sul de Minas Gerais, a soja vem apresentando um crescente aumento em área plantada, visando à produção de grãos tanto para alimentação humana quanto animal (Carvalho et al., 2010). A adoção de práticas conservacionistas no planejamento agrícola, como o uso de variedades de soja que proporcionam maior cobertura vegetal do terreno e que, consequentemente, reduzam o impacto direto das gotas de chuva sobre a superfície do solo, diminuindo as perdas de solo, água, matéria orgânica e nutrientes por erosão hídrica, bem como o desenvolvimento de modelos que estimem esta cobertura vegetal, assumem um papel fundamental no controle da erosão hídrica e na utilização do solo de forma sustentável (Silva et al., 2005).

A cobertura e o manejo do solo (fator $\mathrm{C}$ em modelos de predição de perdas de solo) para uma cultura, em determinado sistema de manejo e local específicos, representam seu efeito conjunto na redução da erosão hídrica. No entanto, esse fator varia amplamente de acordo com a erosividade e erodibilidade, para cada cultura e cultivo do solo (Bertol et al., 2001; Silva et al., 2009).

De acordo com Roloff \& Bertol (1998), o desenvolvimento de um banco de dados relacionado à cobertura vegetal viabilizou o uso de modelos como Soil Loss Estimator for Meridional and Southern África (SLEMSA), Revised Universal Soil Loss Equation (RUSLE), Erosion Productivy Impact Calculator (EPIC) e Water Erosion Prediction Project (WEPP). A determinação da cobertura vegetal é requerida por vários modelos que estimam as perdas de solo, sendo que os mais utilizados no Brasil são o de Wischmeier \& Smith (1958) e Stocking (1985). Esses modelos permitem o monitoramento da cobertura gerada pela cultura a ser avaliada, do início do plantio até a colheita, podendo ser identificado o período de máxima e mínima cobertura do solo proporcionado pela cultura em questão (Souza et al., 2010). Roloff \& Bertol (1998), adaptando metodologia de Stocking (1988), também desenvolveram modelo matemático para cálculo do fator cobertura (fator C), no entanto, esse método ainda é pouco utilizado no Brasil. De acordo com Hudson (1973), a dificuldade na obtenção do fator C está em estabelecer este valor para um número infinito de possíveis combinações, culturas, rotações, sucessões e outras práticas de manejo, nas quais as perdas de solo devem ser medidas. Conforme Maria (1997), os resultados de cobertura vegetal obtidos para certa combinação de fatores, cultura, manejo e rotação têm utilização restrita a essas condições.

Diversos fatores climáticos e de solo controlam o grau de cobertura vegetal proporcionado pelo dossel das culturas. Para que os modelos estatísticos possam ser usados, é necessário que a cobertura vegetal possa ser estimada de maneira simplificada (Roloff \& Bertol, 1998). De acordo com Kiniry et al. (1992) e Arnold et al. (1995), o uso de dados climáticos para estimativas da cobertura vegetal é de grande importância, uma vez que estas informações são de fácil obtenção em estações metereológicas.

Dessa forma, neste estudo, objetivou-se determinar o índice de cobertura vegetal e desenvolver modelos para sua estimativa para a cultura da soja, usando atributos climáticos no período de chuvas intensas no Sul de Minas Gerais.

\section{MATERIAL E MÉTODOS}

O experimento foi realizado entre novembro de 1999 e maio de 2000 na área experimental do Departamento de Ciência do Solo da Universidade Federal de Lavras - UFLA, região Sul do Estado de Minas Gerais, nas coordenadas $21^{\circ} 14^{\prime}$ de latitude Sul e $45^{\circ} 00^{\prime}$ de longitude Oeste. A altitude média da área é de 918,84 m. O clima é enquadrado, de acordo com a classificação de Köppen, como Cwa, com precipitação média anual de $1529,7 \mathrm{~mm}$. A temperatura média anual é de 19,4 C (Ometto, 1981; Brasil, 1992). O solo no local do experimento é um Latossolo Vermelho Distroférrico típico (LVdf) (Empresa Brasileira de Pesquisa Agropecuária Embrapa, 2006).

Para avaliação da cobertura vegetal (CV) foi utilizada a metodologia descrita por Stocking (1988), na qual o observador mira verticalmente por um par de orifícios situados nas barras superiores do aparelho. Quando o solo observado é desnudo ou apenas com restos de vegetação, registra-se " 0 ". Caso o campo de visão seja parcialmente coberto por vegetação registra-se " 0,5 ”. E se é avistada vegetação, registra-se "1". As leituras foram realizadas transversalmente às linhas de plantio e de forma aleatória nas parcelas das cultivares de soja, sendo realizadas essas medições semanalmente. Admitindo-se que a própria posição da cobertura seja casual, foram realizadas 30 leituras casuais no campo, de acordo com a metodologia.

Para a avaliação do índice de cobertura vegetal (CV) foi utilizada a seguinte equação:

$$
\mathrm{CV}(\%)=\frac{\text { Soma das coberturas lidas }}{\text { Número de leituras }} \times 100
$$

A cobertura vegetal foi avaliada em seis estádios da cultura, representando condições uniformes de recobrimento do solo e de efeitos de manejo, a saber: D do preparo do solo ao plantio (descoberto); PL - do plantio 
até 35 dias depois (pós-plantio); 1 - dos 35 dias aos 60 dias após o plantio (estabelecimento); 2 - dos 60 aos 80 dias depois do plantio (desenvolvimento); 3 - dos 80 dias do plantio até a colheita (maturação); e 4 - da colheita ao novo preparo do solo (resíduo).

Foram avaliadas vinte e oito cultivares de soja, em plantio convencional, com potencial para cultivo no Sul de Minas Gerais. O espaçamento adotado foi de $0,5 \mathrm{~m}$ entre linhas com densidade populacional de 20 plantas por metro linear, com stand de 400.000 plantas por hectare. A adubação de plantio foi realizada utilizando-se $400 \mathrm{~kg} \mathrm{ha}^{-1}$ do formulado 4-30-16.

Os atributos climáticos utilizados para desenvolvimento dos modelos foram: temperatura mínima (Tmin), temperatura média (Tmed), temperatura máxima (Tmax), precipitação (PREC), horas de radiação solar incidente na cultura (INS), umidade relativa do ar (UR) e evapotranspiração (EP). Assim, procurou-se relacionar a CV com os valores acumulados dos atributos climatológicos.

Para o estudo de erosividade $\left(\mathrm{EI}_{30}\right)$, foram utilizados dados pluviométricos obtidos junto à Estação Climatológica Principal de Lavras, localizada no campus da UFLA, correspondentes aos meses de novembro de 1999 a maio de 2000, com dados gerados a cada 5 minutos. A partir das precipitações, foram calculadas energias cinéticas totais das chuvas para cada evento (Silva et al., 2009). Foram consideradas chuvas individuais aquelas separadas por mais de 6 horas. As chuvas com valores menores que $10 \mathrm{~mm}$, com intensidade máxima menor que $24 \mathrm{~mm} \mathrm{~h}^{-1}$, em 15 minutos, ou com energia cinética menor que 3,6 MJ, foram consideradas não erosivas (Maria, 1994). Para o cálculo da energia cinética (Ec), utilizou-se a equação proposta por Wagner \& Massambani (1988):

\section{$\mathrm{Ec}=0,153+0,0645 \log \mathrm{I}$}

Onde: Ec é a energia cinética da chuva $\left(\mathrm{MJ} \mathrm{ha}^{-1} \mathrm{~mm}^{-1}\right)$ e I é a intensidade da chuva $\left(\mathrm{mm} \mathrm{h}^{-1}\right)$.

$\mathrm{O}$ índice $\mathrm{EI}_{30}$ foi obtido a partir da multiplicação da energia cinética total pela máxima intensidade verificada em um período de 30 minutos consecutivos $\left(\mathrm{I}_{30}\right)$, segundo Wischmeier \& Smith (1958).

Para a obtenção dos modelos usou-se o programa estatístico "Statistical Analysis System" (SAS Institute, 1995), sendo os mesmos ajustados por meio de análise de regressão linear múltipla, pelo processo "backward". O nível mínimo de significância pelo teste t para exclusão de uma variável foi de $5 \%$, o qual foi estipulado para preservação do nível global de significância do modelo, onde se eliminou em cada passo a variável que não apresentava significância $(\mathrm{P}>0,05)$. Esse procedimento foi repetido até que não houvesse mais variáveis nãosignificativas.

\section{RESULTADOS E DISCUSSÃO}

O caráter morfoagronômico, representado pelo hábito de crescimento, altura de plantas, altura de inserção da $1^{\text {a }}$ vagem e variáveis de rendimento (massa total, palha e grãos) para as cultivares de soja avaliadas, pode ser verificado na Tabela 1, bem como a distribuição dos valores mensais de erosividade e da precipitação pluvial, no período de estudo (Figura 1).

Analisando os valores médios das variáveis apresentadas na Tabela 1, observa-se que os valores apresentaram uma amplitude de variação de 39,7 a 129,3 cm; 10,7 a 33,3 cm; 2916,7 a 8583, $3 \mathrm{~kg} \mathrm{ha}^{-1}$; 1372,7 a 3592,3 $\mathrm{kg} \mathrm{ha}^{-1}$ e 1413,0 a $3081 \mathrm{~kg} \mathrm{ha}^{-1}$ para as variáveis, altura de plantas, altura de inserção da 1a vagem e variáveis de rendimento (massa total, palha e rendimento de grãos), respectivamente. Entre cultivares, em termos de valores absolutos de rendimento de grãos, a mais eficiente foi a $\mathrm{M}$. Soy 108 e a menos a Tucano, em associação com as características morfoagronômicas dessas cultivares.

$\mathrm{Na}$ Figura 1, podem ser verificadas as distribuições dos valores mensais da precipitação pluvial e do índice de erosividade, $\mathrm{EI}_{30}$, para a região de Lavras, $\mathrm{MG}$, no período de estudo. Observa-se que os valores máximos estão concentrados no período inicial (janeiro a março) e final (dezembro) do ano, com a precipitação variando de 4 a 460 $\mathrm{mm}$, para os meses de maio e janeiro, respectivamente. Resultados semelhantes foram observados por Val et al. (1986) e mais recentemente por Silva et al. (2009).

Em relação aos valores do índice de erosividade mensal, observa-se que os valores máximos estão concentrados no período inicial (janeiro a março) e final (dezembro) do ano, com erosividade variando de 1 a $3.429 \mathrm{MJ} \mathrm{mm} \mathrm{ha}^{-1} \mathrm{~h}^{-1} \mathrm{mês}^{-1}$, para os meses de maio e janeiro, respectivamente. Esse comportamento também tem sido observado por outros pesquisadores (Carvalho et al., 1989; Silva et al., 2009).

De acordo com Rufino (1986), valores de erosividade mensal acima de $500 \mathrm{MJ} \mathrm{mm} \mathrm{ha}^{-1} \mathrm{~h}^{-1}$ mês $^{-1}$ podem ser considerados críticos. No período de estudo, em vários meses foram observados valores muito acima desse valor crítico, evidenciando a necessidade de se evitar que eventos de chuvas com alto poder erosivo coincidam com o solo descoberto. Dessa forma, o conhecimento do potencial de cobertura do solo oferecido pela cultura, bem como as épocas do ano em que os riscos de erosão são mais eminentes, são de fundamental importância para o planejamento das atividades de uso e manejo do solo. 
Na Tabela 2, estão apresentados os modelos ajustados de cobertura vegetal ao longo do ciclo da cultura, bem como os coeficientes de determinação e índice de cobertura vegetal para as cultivares de soja estudadas, aos 60 dias após o plantio. Foram estabelecidos modelos relacionando a cobertura vegetal $(\mathrm{CV})$ e os dias decorridos após o plantio (D), sendo verificado o melhor ajuste para o modelo quadrático, que apresentou significância a $1 \%$ e alto coeficiente de determinação para a maioria das cultivares estudadas.
Os valores de cobertura vegetal apresentaram uma amplitude de variação de 56 a $83 \%$, sendo a cultivar FT Abyara e BR 162, as que apresentaram o menor e o maior valor, respectivamente. $\mathrm{O}$ modelo ajustado mais adequado para estimativa da cobertura vegetal foi obtido a partir do índice de cobertura das cultivares, bem como de algumas variáveis climáticas (Tabela 3), obtendo-se assim a equação: $\mathrm{CV}=116589,976+0,422$. Tmed $+0,132$. PREC 0,095. UR + 0,000024. Tmed ${ }^{2}, \mathrm{R}^{2}=0,99(\mathrm{P}<0,01)$. Embora não exista relação de causa e efeito entre as variáveis

Tabela 1 - Hábito de crescimento (HC), altura de plantas (AP), altura de inserção da 1ํㅡ vagem (AIV) e rendimento (massa total, palha e grãos) para as cultivares de soja avaliadas. Com exceção do HC, os outros valores representam médias das variáveis envolvidas.

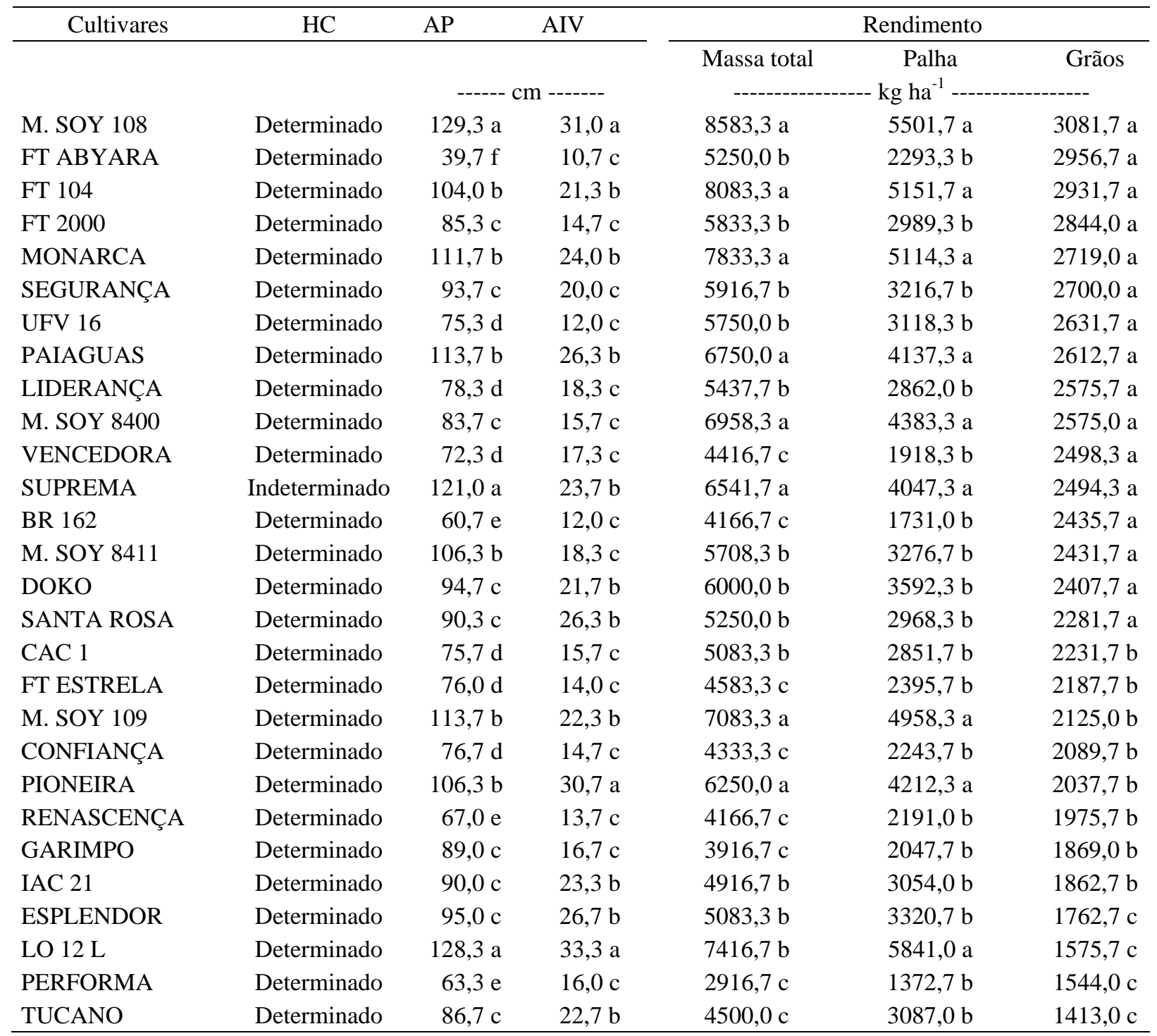

Médias seguidas pela mesma letra na coluna não diferem entre si pelo teste de Scott-Knott ao nível de 5\% de significância. 


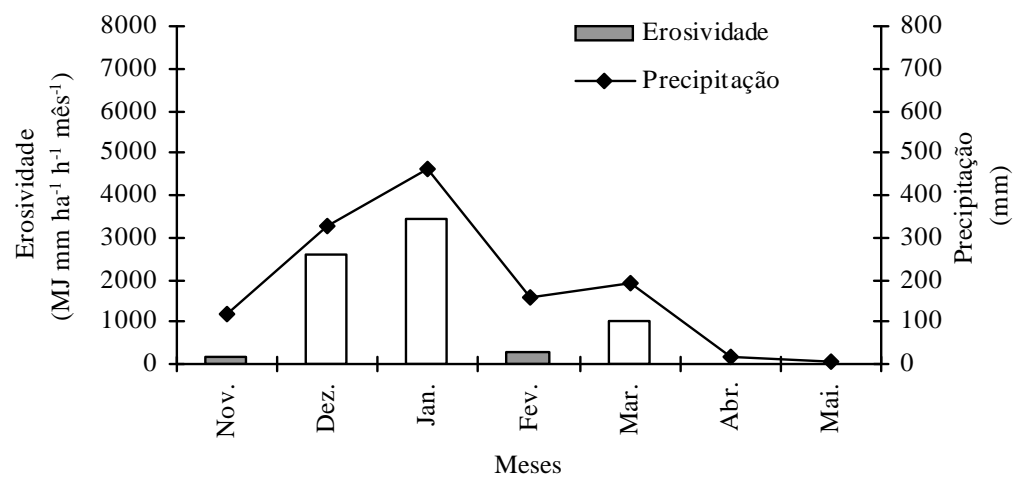

Figura 1 - Distribuição dos valores mensais do índice de erosividade, $\mathrm{EI}_{30}$, e da precipitação pluviométrica para a região de Lavras, MG, no período de novembro de 1999 a maio de 2000.

Tabela 2 - Cultivares de soja avaliados, modelos para a estimativa do índice de cobertura vegetal e valores de índice de cobertura vegetal (CV) aos 60 dias após o plantio da cultura.

\begin{tabular}{llcc}
\hline \multicolumn{1}{c}{ Cultivares } & \multicolumn{1}{c}{ Modelos Quadráticos } & R $(\%)$ \\
\hline M. SOY 108 & CV $=-0,020635 \mathrm{D}^{2}+3,9326 \mathrm{D}-82,14$ & 0,93 & 80 \\
FT ABYARA & $\mathrm{CV}=-0,017319 \mathrm{D}^{2}+3,6514 \mathrm{D}-101,17$ & 0,92 & 56 \\
FT 104 & $\mathrm{CV}=-0,023167 \mathrm{D}^{2}+4,4152 \mathrm{D}-103,45$ & 0,90 & 78 \\
FT 2000 & $\mathrm{CV}=-0,019789 \mathrm{D}^{2}+4,0650 \mathrm{D}-115,15$ & 0,94 & 58 \\
MONARCA & $\mathrm{CV}=-0,021273 \mathrm{D}^{2}+4,1433 \mathrm{D}-95,66$ & 0,92 & 76 \\
SEGURANÇA & $\mathrm{CV}=-0,018893 \mathrm{D}^{2}+3,8214 \mathrm{D}-89,42$ & 0,92 & 72 \\
UFV 16 & $\mathrm{CV}=-0,024796 \mathrm{D}^{2}+4,9623 \mathrm{D}-144,94$ & 0,94 & 64 \\
PAIAGUAS & $\mathrm{CV}=-0,022334 \mathrm{D}^{2}+4,3361 \mathrm{D}-107,15$ & 0,93 & 73 \\
LIDERANÇA & $\mathrm{CV}=-0,017522 \mathrm{D}^{2}+3,5695 \mathrm{D}-79,11$ & 0,92 & 72 \\
M. SOY 8400 & $\mathrm{CV}=-0,019934 \mathrm{D}^{2}+3,9118 \mathrm{D}-91,51$ & 0,94 & 71 \\
VENCEDORA & $\mathrm{CV}=-0,022623 \mathrm{D}^{2}+4,4470 \mathrm{D}-115,32$ & 0,92 & 70 \\
SUPREMA & $\mathrm{CV}=-0,021477 \mathrm{D}^{2}+4,1270 \mathrm{D}-94,85$ & 0,95 & 75 \\
BR 162 & $\mathrm{CV}=-0,038855 \mathrm{D}^{2}+6,4720 \mathrm{D}-165,40$ & 0,89 & 83 \\
M. SOY 8411 & $\mathrm{CV}=-0,022081 \mathrm{D}^{2}+4,2692 \mathrm{D}-99,78$ & 0,91 & 77 \\
DOKO & $\mathrm{CV}=-0,019515 \mathrm{D}^{2}+4,0801 \mathrm{D}-115,49$ & 0,91 & 59 \\
SANTA ROSA & $\mathrm{CV}=-0,023198 \mathrm{D}^{2}+4,3209 \mathrm{D}-96,42$ & 0,97 & 79 \\
CAC 1 & $\mathrm{CV}=-0,019393 \mathrm{D}^{2}+4,1440 \mathrm{D}-121,88$ & 0,94 & 57 \\
FT ESTRELA & $\mathrm{CV}=-0,026783 \mathrm{D}^{2}+4,8198 \mathrm{D}-114,52$ & 0,93 & 78 \\
M. SOY 109 & $\mathrm{CV}=-0,023162 \mathrm{D}^{2}+4,4963 \mathrm{D}-112,17$ & 0,91 & 74 \\
CONFIANÇA & $\mathrm{CV}=-0,028548 \mathrm{D}^{2}+5,1710 \mathrm{D}-136,99$ & 0,90 & 70 \\
PIONEIRA & $\mathrm{CV}=-0,021303 \mathrm{D}^{2}+4,2988 \mathrm{D}-114,23$ & 0,97 & 67 \\
RENASCENÇA & $\mathrm{CV}=-0,032479 \mathrm{D}^{2}+5,7410 \mathrm{D}-154,89$ & 0,87 & 73 \\
GARIMPO & $\mathrm{CV}=-0,021864 \mathrm{D}^{2}+4,3233 \mathrm{D}-111,21$ & 0,91 & 69 \\
IAC 21 & $\mathrm{CV}=-0,018778 \mathrm{D}^{2}+3,8609 \mathrm{D}-98,56$ & 0,94 & 65 \\
ESPLENDOR & $\mathrm{CV}=-0,021787 \mathrm{D}^{2}+4,2404 \mathrm{D}-104,08$ & 0,94 & 72 \\
LO 12 L & $\mathrm{CV}=-0,023019 \mathrm{D}^{2}+4,2450 \mathrm{D}-90,30$ & 0,95 & 82 \\
PERFORMA & $\mathrm{CV}=-0,018253 \mathrm{D}^{2}+3,8111 \mathrm{D}-100,81$ & 0,88 & 62 \\
TUCANO & $\mathrm{CV}=-0,017818 \mathrm{D}^{2}+3,7840 \mathrm{D}-106,04$ & 0,94 & 57 \\
\hline & & & \\
\hline
\end{tabular}

Ciênc. agrotec., Lavras, v. 34, n. 5, p. 1168-1175, set./out., 2010 
Tabela 3 - Valores mínimos, médios e máximos diários das variáveis climáticas estudadas incluídas no modelo para estimativa do índice de cobertura vegetal para a cultura da soja.

\begin{tabular}{lcccc}
\hline \multirow{2}{*}{ Variável } & \multirow{2}{*}{ Símolo } & \multicolumn{3}{c}{ Valor } \\
\cline { 3 - 5 } & & Mínimo & Médio & Máximo \\
\hline Índice de cobertura vegetal $(\%)$ & $\mathrm{CV}$ & 19,3 & 81,9 & 100,0 \\
Temperatura mínima $\left({ }^{\circ} \mathrm{C}\right)$ & $\mathrm{T}_{\min }$ & 13,3 & 17,9 & 20,7 \\
Temperatura média $\left({ }^{\circ} \mathrm{C}\right)$ & $\mathrm{T}_{\operatorname{med}}$ & 15,7 & 22,2 & 25,5 \\
Temperatura máxima $\left({ }^{\circ} \mathrm{C}\right)$ & $\mathrm{T}_{\max }$ & 20,0 & 28,1 & 33,0 \\
Precipitação pluvial $(\mathrm{mm})$ & $\mathrm{PREC}$ & 0,0 & 9,6 & 86,8 \\
Horas de radiação solar $(\mathrm{n})$ & $\mathrm{INS}$ & 0,0 & 6,2 & 13,6 \\
Umidade relativa do ar $(\%)$ & $\mathrm{UR}$ & 54 & 77,0 & 97,0 \\
Evapotranspiração $(\mathrm{mm})$ & $\mathrm{EP}$ & 0,5 & 3,9 & 9,6 \\
\hline
\end{tabular}

selecionadas e a cobertura vegetal, fica claro que a maioria delas tem participação nos processos que governam o desenvolvimento do dossel, com o modelo apresentando elevado coeficiente de determinação.

O comportamento do índice de cobertura vegetal no decorrer do ciclo da cultura, bem como em consonância com seus estádios de desenvolvimento, pode ser observado nas Figuras 2 e 3, respectivamente. Pode-se observar que as cultivares M. Soy 108 e Tucano foram os que obtiveram, respectivamente, os maiores e menores valores de cobertura vegetal. Verifica-se que, após 35 dias da emergência (estádio 1), a cobertura vegetal proporcionada foi superior a $20 \%$ para a cultivar M. Soy 108 e inferior a $20 \%$ para a Tucano.

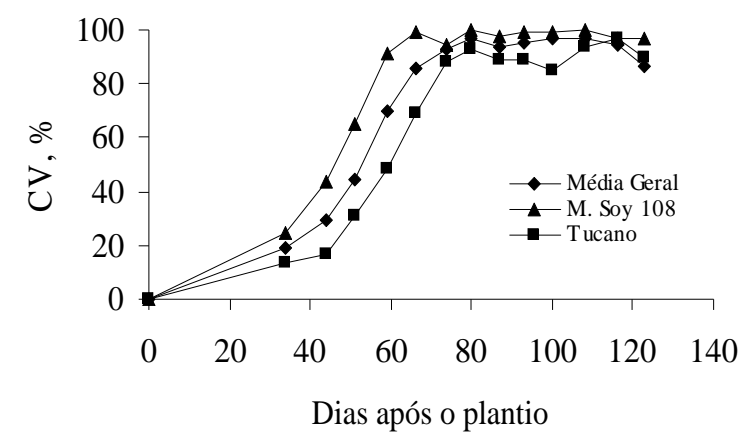

Figura 2 - Evolução da cobertura vegetal (CV) de dois cultivares de soja.

Segundo relatos de literatura, sistemas tidos como conservacionistas apresentam índices de cobertura do solo superiores a $30 \%$ nos períodos de cultivo e pousio (Lopes et al., 1987). Trabalhando com chuva natural em Latossolo Vermelho Distroférrico típico (LVdf), similar ao deste estudo, em Dourados (MS), Hernani et al. (1997) constataram maior eficácia da semeadura direta na palha, (especialmente de gramíneas) em relação aos demais sistemas de manejo do solo na redução da erosão hídrica em todos os estádios de crescimento das culturas de trigo e soja. O plantio direto integrado com pastagem pode ser incluído neste contexto, sendo o plantio direto realizado sobre a pastagem dessecada (cobertura morta). Por melhor que seja a cobertura do solo pela soja, sempre irá ocorrer período de solo descoberto, justamente na época de chuvas mais erosivas, onde a cobertura morta assume papel relevante, até a soja atingir seu índice de área foliar máximo. Esse manejo tem se expandido geograficamente no Sul de Minas Gerais mais recentemente.

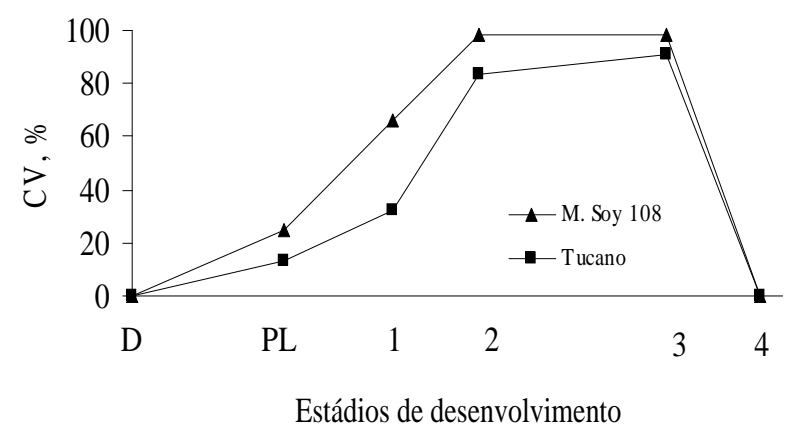

Figura 3 - Cobertura vegetal (CV) de dois cultivares de soja em relação aos seus estádios de desenvolvimento explicitados em Material e Métodos.

\section{CONCLUSÕES}

Entre as cultivares estudadas, considerando a produção de grãos e a cobertura vegetal, as mais eficientes foram BR 162 e M. Soy 108. 
Os resultados obtidos neste estudo poderão subsidiar a escolha das cultivares de soja e o manejo adotado nesta cultura, no Sul de Minas Gerais, tendo em vista a sustentabilidade desta exploração no tocante à erosão hídrica e o crescente aumento de sua área plantada.

\section{REFERÊNCIAS BIBLIOGRÁFICAS}

AMADO, T.J.C.; COGO, N.; LEVIEN, R. Eficácia relativa do manejo do resíduo cultural de soja na redução das perdas de solo por erosão hídrica. Revista Brasileira de Ciência do Solo, Campinas, v.13, n.2, p.251-257, 1989.

ARNOLD, J.G.; WELTZ, M.A.; ALBERTS, E.E.; FLANAGAN, D.C. Plant growth component. In: LANE, L.J.; NEARING, M.A. (Eds.). Water erosion prediction project: hillslope profire model documentation. West Lafayette: USDA-ARS, 1995. chap.8, p.1-41.

BERTOL, I.; SCHICK, J.; BATISTELA, O. Razão de perdas de solo e fator para as culturas de soja e trigo em três sistemas de preparo em um cambissolo húmico alumínico. Revista Brasileira de Ciência do Solo, Viçosa, v.25, n.2, p.451-461, 2001.

BRASIL. Ministério da Agricultura e Reforma agrária. Normais climatológicas: 1961-1990. Brasília: Secretaria Nacional de Irrigação, 1992. 84p.

CARVALHO, E.R.; REZENDE, P.M.; OGOSHI, F.G.A.; BOTREL, E.P.; ALCANTARA, H.P.; SANTOS, J.P.

Desempenho de cultivares de soja [Glycine max (L.) Merrill] em cultivo de verão no Sul de Minas Gerais. Ciência e Agrotecnologia, Lavras, v.34, n.4, p.892-899, 2010.

CARVALHO, M.P.; LOMBARDI NETO, F.; VASQUES FILHO, J.; CATANEO, A. Erosividade da chuva de Mococa (SP) analisada pelo índice $\mathrm{EI}_{30}$. Revista Brasileira de Ciência do Solo, Campinas, v.13, n.2, p.243-249, 1989.

EMPRESA BRASILEIRA DE PESQUISA AGROPECUÁRIA. Sistema brasileiro de classificação de solos. Rio de Janeiro, 2006. 306p.

HERNANI, L.C.; SALTON, J.C.; FABRÍCIO, A.C.; DEDECEK, R.; ALVES JÚNIOR, M. Perdas por erosão e rendimentos de soja e trigo em diferentes sistemas de preparo de um Latossolo Roxo de Dourados (MS).
Revista Brasileira de Ciência do Solo, Campinas, v.21, n.3, p.667-676, 1997.

HUDSON, N.W. Soil conservation. 2.ed. Ithaca: Cornell University, 1973. 320p.

KINIRY, J.R.; WILLIAMS, J.R.; GASSMAN, P.W.; DEBACKE, P. General, process-oriented model for two competing plant species. Transactions American Society Agricultural Engineering, v.35, p.801-810, 1992.

LOPES, P.R.C.; COGO, N.P.; LEVIEN, R. Eficácia relativa de tipos e quantidade de resíduos culturais espalhados uniformemente sobre o solo na redução da erosão hídrica. Revista Brasileira de Ciência do Solo, Campinas, v.11, p.71-75, 1987

MARIA, I.C. de; LOMBARDI NETO, F. Razão de perdas de solo e fator $\mathrm{C}$ para sistemas de manejo da cultura do milho. Revista Brasileira de Ciência do Solo, Campinas, v.21, n.2, p.263-270, 1997.

MARIA, I.C. de. Cálculo da erosividade da chuva. In: INSTITUTO AGRONÔMICO DE CAMPINAS. Manual de programas de processamento de dados de campo e de laboratório para fins de experimentação em conservação do solo. Campinas, 1994.

OMETTO, J.C. Bioclimatologia vegetal. São Paulo: Ceres, 1981. 400p.

ROLOFF, G.; BERTOL, O.J. Método para estimativa da cobertura do solo e da altura do dossel de algumas culturas de verão. Revista Brasileira de Ciência do Solo, Viçosa, v.22, n.2, p.319-327, 1998.

RUFINO, R.L. Avaliação do potencial erosivo da chuva para o Estado do Paraná: segunda aproximação. Revista Brasileira de Ciência do Solo, Campinas, v.10, n.3, p.279-281, 1986.

SAS INSTITUTE. SAS language and procedures: usage version 6. Cary, 1985. 373p.

SILVA, A.M.; SILVA, M.L.N.; CURI, N.; AVANZI, J.C.; FERREIRA, M.M. E rosividade da chuva e erodibilidade de Cambissolo e Latossolo na região de Lavras, Sul de Minas Gerais. Revista Brasileira de Ciência do Solo, Viçosa, v.33, n.6, p.1811-1820, 2009. 
SILVA, A.M.; SILVA, M.L.N.; CURI, N.; LIMA, J.M.; AVANZI, J.C.; FERREIRA, M.M. Perdas de solo, água, nutrientes e carbono orgânico em Cambissolo e Latossolo sob chuva natural. Pesquisa Agropecuária Brasileira, Brasília, v.40, n.12, p.1233-1230, 2005.

SOUZA, F.S.; SILVA, M.L.N.; CURI, N.; AVANZI, J.C.; PINHO, R.G.; LIMA, G.C. Índice de cobertura vegetal pela cultura do milho no período de chuvas intensas no Sul de Minas Gerais. Ciência e Agrotecnologia, Lavras, v.34, n.2, p.345-351, 2010.

STOCKING, M.A. Assessing vegetative cover and management effects. In: LAL, R. (Ed.). Soil erosion research methods. Ankeny: Soil and Water

Conservation Society, 1988. p.163-185.

STOCKING, M.A. Modelagem de perdas de solo: sugestões para uma aproximação brasileira. Brasília: PNUD, 1985. 92p. Projeto PNUD/FAO, BRA/82011.
VAL, L.A.; BAHIA, V.G.; FREIRE, J.C.; DIAS JÚNIOR, M.S. Erosividade das chuvas em Lavras, MG. Ciência e Prática, Lavras, v.10, n.2, p.199-209, 1986.

WAGNER, C.S.; MASSAMBINI, O. Análise da relação intensidade de chuva: energia de Wischmeier \& Smith e sua aplicabilidade à região de São Paulo. Revista Brasileira de Ciência do Solo, Campinas, v.12, n.3, p.197-203, 1988.

WISCHMEIER, W.H.; SMITH, D.D. Predicting rainfall erosion losses: a guide to conservation planning. Washington: USDA, 1958. 58p. (Agriculture Hand-book, 537).

WISCHMEIER, W.H.; SMITH, D.D. Rainfall energy and its relationships to soil loss. Transactions of the American Geophysical Union, Washington, v.39, n.2, p.285-291, 1958. 